\title{
ARTICLE OPEN \\ Observation of topological Uhlmann phases with superconducting qubits
}

\author{
O. Viyuela ${ }^{1,2,3}$, A. Rivas ${ }^{1}$, S. Gasparinetti ${ }^{4}$, A. Wallraff ${ }^{4}{ }^{4}$, S. Filipp ${ }^{5}$ and M. A. Martin-Delgado ${ }^{1}$
}

Topological insulators and superconductors at finite temperature can be characterized by the topological Uhlmann phase. However, a direct experimental measurement of this invariant has remained elusive in condensed matter systems. Here, we report a measurement of the topological Uhlmann phase for a topological insulator simulated by a system of entangled qubits in the IBM Quantum Experience platform. By making use of ancilla states, otherwise unobservable phases carrying topological information about the system become accessible, enabling the experimental determination of a complete phase diagram including environmental effects. We employ a state-independent measurement protocol which does not involve prior knowledge of the system state. The proposed measurement scheme is extensible to interacting particles and topological models with a large number of bands.

npj Quantum Information (2018)4:10; doi:10.1038/s41534-017-0056-9

\section{INTRODUCTION}

The search for topological phases in condensed matter ${ }^{1-8}$ has triggered an experimental race to detect and measure topological phenomena in a wide variety of quantum simulation experiments. ${ }^{9-15}$ In quantum simulators the phase of the wave function can be accessed directly, opening a whole new way to observe topological properties ${ }^{9,11,16}$ beyond the realm of traditional condensed matter scenarios. These quantum phases are very fragile, but when controlled and mastered, they can produce very powerful computational systems like a quantum computer. ${ }^{17,18}$ The Berry phase ${ }^{19}$ is a special instance of quantum phase, that is purely geometrical ${ }^{20}$ and independent of dynamical contributions during the time evolution of a quantum system. In addition, if that phase is invariant under deformations of the path traced out by the system during its evolution, it becomes topological. Topological Berry phases have also acquired a great relevance in condensed matter systems. The now very active field of topological insulators (TIs) and superconductors (TSCs) $)^{1-3}$ ultimately owes its topological character to Berry phases ${ }^{21}$ associated to the special band structure of these exotic materials.

However, if the interaction of a TI or a TSC with its environment is not negligible, the effect of the external noise in the form of, e.g., thermal fluctuations, makes these quantum phases very fragile, $^{22-34}$ and they may not even be well defined. For the Berry phase acquired by a pure state, this problem has been successfully adressed for one-dimensional systems ${ }^{35}$ and extended to twodimensions later. ${ }^{36-38}$ The key concept behind this theoretical characterization is the notion of "Uhlmann phase", ${ }^{39-47}$ a natural extension of the Berry phase for density matrices. In analogy to the Berry phase, when the Uhlmann phase for mixed states remains invariant under deformations, it becomes topological.

Although this phase is gauge invariant and thus, in principle, observable, a fundamental question remains: how to measure a topological Uhlmann phase in a physical system? To this end, we employ an ancillary system as a part of the measurement apparatus. By encoding the temperature (or mixedness) of the system in the entanglement with the ancilla, we find that the Uhlmann phase appears as a relative phase that can be retrieved by interferometric techniques. The difficulty with this type of measurement is that it requires a high level of control over the environmental degrees of freedom, beyond the reach of condensed matter experiments. On the contrary, this situation is especially well-suited for a quantum simulation scenario.

Specifically, in this work we report: (i) the measurement of the topological Uhlmann phase on a quantum simulator based on superconducting qubits, ${ }^{48-50}$ in which we have direct control over both system and ancilla, and (ii) the computation of the topological phase diagram for qubits with an arbitrary noise degree. A summary and a comparison with pure state topological measures are shown in Fig. 1. In addition, we construct a state independent protocol that detects whether a given mixed state is topological in the Uhlmann sense. Our proposal also provides a quantum simulation of the Alll class $5^{51,52}$ of TIs (those with chiral symmetry) in the presence of disturbing external noise. Other cases of two-dimensional Tls, TSCs and interacting systems can also be addressed by appropriate modifications as mentioned in the conclusions.

\section{RESULTS}

Topological Uhlmann phase for qubits

We briefly present the main ideas of the Uhlmann approach for a two-band model of Tls and TSCs simulated with a qubit. Let $\left.\theta(t)\right|_{t=0} ^{1}$ define a closed trajectory along a family of single qubit

\footnotetext{
${ }^{1}$ Departamento de Física Teórica I, Universidad Complutense, 28040 Madrid, Spain; ${ }^{2}$ Department of Physics, Massachusetts Institute of Technology, Cambridge, MA 02139, USA; ${ }^{3}$ Department of Physics, Harvard University, Cambridge, MA 02318, USA; ${ }^{4}$ Department of Physics, ETH Zurich, CH-8093 Zurich, Switzerland and ${ }^{5}$ BMM Research - Zurich, 8803 Rueschlikon, Switzerland

Correspondence: O. Viyuela (oviyuela@mit.edu)
}

Received: 5 September 2017 Revised: 19 December 2017 Accepted: 27 December 2017

Published online: 12 February 2018 


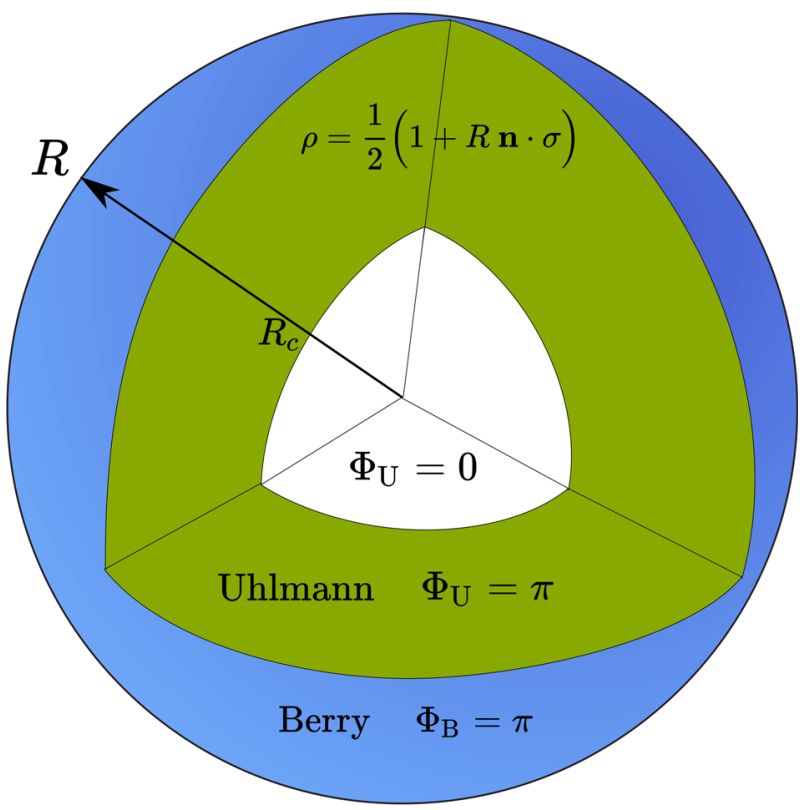

Fig. 1 Topological measures for a single qubit in a mixed state $\rho=$ $(1-r)|1\rangle\langle 1|+r| 0\rangle\langle 0|=\frac{1}{2}(+R \mathbf{n} \cdot \sigma)$ in the Bloch sphere representation. The mixedness parameter $r$ between states $|1\rangle$ and $|0\rangle$ is encoded into the degree of mixedness $R=|2 r-1|$. We compute the Berry $\Phi_{B}$ and Uhlmann $\Phi_{U}$ phases for non-trivial topological regimes. If $r \notin\{1,0\}$ or equivalently $R<1$, then only $\Phi_{U}$ is well defined and highlights a non-trivial topological phase $\left(\Phi_{U}=\pi\right)$, provided that $R>R_{\mathrm{c}}$. Here, $R_{\mathrm{c}}$ denotes the critical amount of noise that the system can withstand while remaining topological

density matrices parametrized by $\theta$,

$\rho_{\theta}=(1-r)\left|0_{\theta}\right\rangle\left\langle 0_{\theta}|+r| 1_{\theta}\right\rangle\left\langle 1_{\theta}\right|$,

where $r$ stands for the mixedness parameter between the $\theta$ dependent eigenstates $\left|1_{\theta}\right\rangle$ and $\left|0_{\theta}\right\rangle$, e.g., that of a transmon qubit. $^{53}$ The mixed state $\rho_{\theta}$ can be seen as a "part" of a state vector $\left|\Psi_{\theta}\right\rangle$ in an enlarged Hilbert space $\mathcal{H}=\mathcal{H}_{S} \otimes \mathcal{H}_{A}$, where $S$ stands for system and $A$ for the ancilla degrees of freedom with $\operatorname{dim} \mathcal{H}_{A} \geq \operatorname{dim} \mathcal{H}_{S}$. The state vector $\left|\Psi_{\theta}\right\rangle$ is a so-called purification of $\rho_{\theta}=\operatorname{Tr}_{A}\left(\left|\Psi_{\theta}\right\rangle\left\langle\Psi_{\theta}\right|\right)$, where $\operatorname{Tr}_{\mathrm{A}}$ performs the partial trace over the ancilla. There is an infinite number of purifications for every single density matrix, specifically $\left(\square \otimes U_{A}\right)\left|\Psi_{\theta}\right\rangle$ for any unitary $U_{\mathrm{A}}$ acting on the ancilla purifies the same mixed state as $\left|\Psi_{\theta}\right\rangle$. Hence, for a family of density matrices $\rho_{\theta}$, there are several sets of purifications $\left|\Psi_{\theta}\right\rangle$ according to a $U(n)$ gauge freedom. This generalizes the standard $U(1)$ gauge (phase) freedom of state vectors describing quantum pure states to the general case of density matrices.

Along a trajectory $\left.\theta(t)\right|_{t=0} ^{1}$ for $\rho_{\theta}$ the induced purification evolution (system qubit $S$ and ancilla qubit $A$ ) can be written as

$$
\begin{aligned}
\left|\Psi_{\theta(t)}\right\rangle= & \sqrt{1-r} U_{S}(t)|0\rangle_{S} \otimes U_{A}(t)|0\rangle_{A}+ \\
& +\sqrt{r} U_{S}(t)|1\rangle_{S} \otimes U_{A}(t)|1\rangle_{A},
\end{aligned}
$$

where $|0\rangle=\left(\begin{array}{l}1 \\ 0\end{array}\right)$ and $|1\rangle=\left(\begin{array}{l}0 \\ 1\end{array}\right)$ is the standard qubit basis, and $U_{S}(t)$ is a unitary matrix determined by the $\theta$-dependence. Moreover the arbitrary unitaries $U_{\mathrm{A}}(t)$ can be selected to fulfill the so-called Uhlmann parallel transport condition. Namely, analogously to the standard Berry case, the Uhlmann parallel transport requires that the distance between two infinitesimally close purifications $\|\left|\Psi_{\theta(t+d t)}\right\rangle-\left|\Psi_{\theta(t)}\right\rangle \|^{2}$ reaches a minimum value (which leads to removing the relative infinitesimal "phase" between purifications). ${ }^{39}$ Physically, this condition ensures that the accumulated quantum phase (the so-called Uhlmann phase $\Phi_{U}$ ) along the trajectory is purely geometrical, that is, without dynamical contributions. This is a source of robustness, since variations on the transport velocity will not change the resulting phase.

Next, we consider the Hamiltonian of a two-band TI in the Alll chiral-unitary class, ${ }_{t}^{51,52} H=\sum_{k} \Psi_{k}^{\dagger} H_{k} \Psi_{k}$, in the spinor representation $\Psi_{k}=\left(\hat{a}_{k}, \hat{b}_{k}\right)^{t}$ where $\hat{a}_{k}$ and $\hat{b}_{k}$ stands for two species of fermionic operators. The one-particle Hamiltonian is

$$
\begin{gathered}
H_{k}=\frac{G_{k}}{2} \boldsymbol{n}_{k} \cdot \sigma, \\
\boldsymbol{n}_{k}=\frac{2}{G_{k}}(\sin k, 0, M+\cos k), \\
G_{k}=2 \sqrt{1+M^{2}+2 M \cos k .}
\end{gathered}
$$

where $G_{k}$ represents the actual gap between the valence and conduction bands in the $\mathrm{Tl}$, and $\boldsymbol{n}_{k}$ is a unit vector called winding vector. ${ }^{35}$ We now map the crystalline momentum $k$ of the $\mathrm{TI}^{1-3}$ to a tunable time-dependent paramenter $\theta$ of the quantum simulator. When invoking the rotating wave approximation this model also describes, e.g., the dynamics of a driven transmon qubit. $^{14,16}$ The detuning $\Delta=2(\cos \theta+M)$ between qubit and drive is parametrized in terms of $\theta$ and a hopping amplitude $M$, whereas the coupling strength between the qubit and the incident microwave field is given by $\Omega=2 \sin \theta$.

The non-trivial topology of pure quantum states $(r \in\{0,1\})$ of this class of topological materials can be witnessed by the winding number. This is defined as the angle swept out by $\boldsymbol{n}_{\theta}$ as $\theta$ varies from 0 to $2 \pi$, namely,

$\omega_{1}:=\frac{1}{2 \pi} \oint\left(\frac{\partial_{\theta} n_{\theta}^{x}}{n_{\theta}^{z}}\right) d \theta$.

Then, using Eqs. (3) and (4), the system is topological $\left(\omega_{1}=1\right)$ when the hopping amplitude is less than unity $(M<1)$ and trivial $\left(\omega_{1}=0\right)$ if $M>1$. In fact, the topological phase diagram coincides with the one given by the Berry phase acquired by the "ground" state $|0\rangle_{\theta}$ (or the "excited" state $|1\rangle_{\theta}$ ) of Hamiltonian (3) when $\theta$ varies from 0 to $2 \pi$, (see Supplementary Note 2).

The computation of the unitary $U_{S}$ in Eq. (2) for a transportation in time of $\theta$ according to the Hamiltonian (3) yields

$U_{S}(t)=e^{-i \int_{0}^{t} h\left(t^{\prime}\right) d t^{\prime} \sigma_{y}}$,

with $h(t):=\frac{\partial_{t} n_{t}^{x}}{2 n^{2}}$. This implements the eigenstate transport $\left|1_{\theta(t)}\right\rangle=U_{S}(t)|1\rangle$ and $\left|0_{\theta(t)}\right\rangle=U_{S}(t)|0\rangle$. In addition, we can consider a similar form for the unitary $U_{\mathrm{A}}$ in Eq. (2),

$U_{A}(t)=\left[U_{S}(t)\right]^{p_{a}}=e^{-i \int_{0}^{t} p_{a} h\left(t^{\prime}\right) d t^{\prime} \boldsymbol{\sigma}_{y}}$,

where the parameter $p_{a} \in[0,1]$ is defined as an ancillary "weight". We find that the Uhlmann parallel transport condition is satisfied for $p_{a}=p_{r}:=2 \sqrt{r(1-r)}$. The detailed technical derivation is provided in Supplementary Notes 1 and 2.

Now, from Eq. (2) it is possible to define the relative phase $\Phi_{M}$ between the initial $\left|\Psi_{\theta(0)}\right\rangle$ and the final state, i.e., $\left|\Psi_{\theta\left(t_{f}\right)}\right\rangle$. For Hamiltonian (3), density matrix (1) and purification (2), we find

$$
\begin{aligned}
\Phi_{M} & :=\arg \left[\left\langle\Psi_{\theta(0)} \mid \Psi_{\theta\left(t_{f}\right)}\right\rangle\right]= \\
= & \arg \left[\cos \left(I_{0}^{t_{f}}\right) \cos \left(p_{a} I_{0}^{t_{f}}\right)+p_{r} \sin \left(I_{0}^{t_{f}}\right) \sin \left(p_{a} I_{0}^{t_{f}}\right)\right],
\end{aligned}
$$

where $l_{t_{0}}^{t_{f}}:=\int_{t_{0}}^{t_{f}} h\left(t^{\prime}\right) d t^{\prime}$. As commented before, by assuming $p_{a}=p_{r}:=2 \sqrt{r(1-r)}$, the purification precisely follows Uhlmann parallel transport and the relative phase $\Phi_{M}$ becomes the Uhlmann phase $\Phi_{U}$ associated to the trajectory. For a closed path $t_{\mathrm{f}}=1$, the integral $I_{0}^{1}=\pi \omega_{1}=\Phi_{B}$ becomes the topological Berry phase. In that case, the Uhlmann phase simplifies to

$\Phi_{U}=\arg \left\{\cos \left[\left(1-2 p_{r}\right) \pi \omega_{1}\right]\right\}$.

We can now deduce the topological properties of these phases in the presence of external noise, as measured by the parameter $r$ 


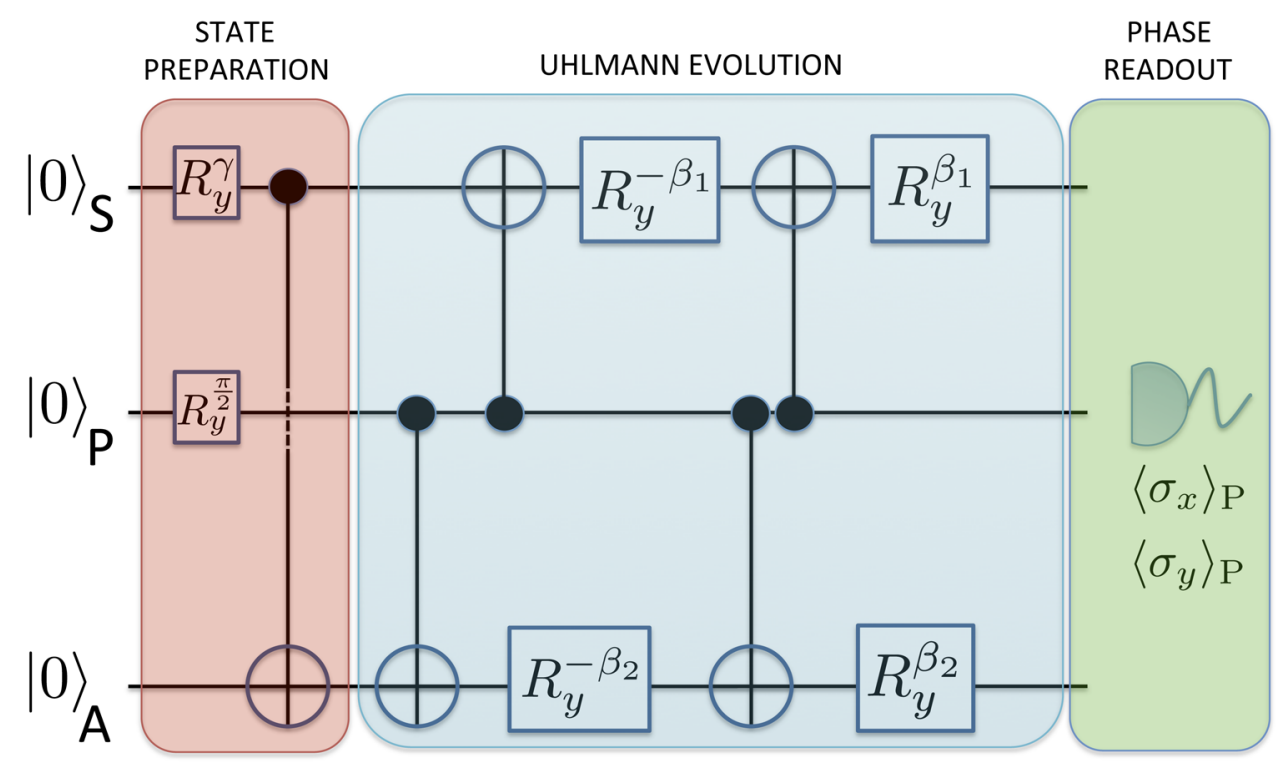

Fig. 2 Circuit diagram to measure the topological Uhlmann phase, e.g., with superconducting circuits as explained in the text. The circuit represents the decomposition of the bi-local unitary evolution $U_{\mathrm{S}}(t) \otimes U_{\mathrm{A}}(t)$, defined in Eq. (5), into elementary single and two-qubit CNOT gates. ${ }^{18}$ The gate $R_{y}^{\gamma}$ represents a single qubit rotation about the $y$-axis for an angle $\gamma=2 \arccos \sqrt{1-r}$, and the angles $\beta_{1}$ and $\beta_{2}$ appear in Eq. (7)

[Eq. (1)]. This is depicted in Fig. 1. Namely, if $M>1$ then $\omega_{1}=0$, and $\Phi_{\mathrm{U}}=0$ (trivial phase) for every mixedness parameter $r$. If $M<1$ then $\omega_{1}=1$ and one obtains $\Phi_{U}=\arg [-\cos (2 \pi \sqrt{r(1-r)})]$. If the state is pure $(r=0)$, then $\Phi_{U}^{0}=\pi$, recovering the same topological phase given by the winding number and the Berry phase. However, for $r \neq 0$ there are critical values of the mixedness $r_{c}$ at which the Uhlmann phase, according to Eq. (8), jumps from $\pi$ to zero (see Fig. 1). The first $r_{c 1}=\frac{1}{4}(2-\sqrt{3}) \approx 0.067$ signals the mixedness at which the system loses the topological character of the ground state. Moreover, there exists another $r_{c 2}=1-r_{c 1}$ at which the system becomes topological again due to the topological character of the excited state $(r \rightarrow 1)$. Notice that at $r=1$ the system becomes a pure state again (the excited state), which is also topologically non-trivial according to the Berry phase. Actually, provided that the weight $p_{r}<p_{r=r_{c 1(2)}}=0.5$, the system is topological in the Uhlmann sense as long as $M<1$. This reentrance in the topological phase at $r_{c 2}$ was absent in previous works. ${ }^{35-37}$

\section{Experimental realization}

Measuring the topological Uhlmann phase is a very challenging task since its definition in terms of purifications implies precise control over auxiliary/environmental degrees of freedom (the ancilla). In an experiment, we therefore include an extra ancilla qubit representing the environment. We also include a third qubit acting as a probe system $P$, such that by measuring qubit $P$ we retrieve the accumulated phase by means of interferometric techniques. The measurement protocol is described in Fig. 2:

Step 1. Following Eq. (2), we prepare the initial state $\left|\Psi_{\theta(0)}\right\rangle \otimes$ $|0\rangle_{P}$ (red block of Fig. 2) using single qubit rotations $R_{y}^{\gamma}$ about the $y$-axis for an angle $\gamma=2 \operatorname{arcos} \sqrt{1-r}$ and a two-qubit controlled not gate. For superconducting qubits, the latter can be performed, e.g., by implementing a controlled phase gate for frequencytunable transmons ${ }^{54}$ or by a cross-resonance gate. ${ }^{55}$

Step 2. We apply the bi-local unitary $U_{S}(t) \otimes U_{A}(t)$ on $S \otimes A$ conditional to the state of the probe $P$. This is accomplished by single qubit rotations about an angle $\beta_{1}$ or $\beta_{2}$, determined by $h(t)$ and $p_{a}$ (blue block of Fig. 2), and two-qubit gates. This decomposition is based on the fact that any controlled unitary gate can be always decomposed as a product of unitary single- qubit gates and two-qubit CNOT gates. ${ }^{18}$ Figure 2 shows the final result after the decomposition of the Uhlmann transport, conditional to the probe qubit $\mathrm{P}$, is performed. As a result, the three qubits $\{S, A, P\}$ are in the superposition

$$
|\Phi\rangle_{S A P}=\frac{1}{\sqrt{2}}\left(\left|\Psi_{\theta(0)}\right\rangle \otimes|0\rangle_{P}+\left|\Psi_{\theta\left(t_{f}\right)}\right\rangle \otimes|1\rangle_{P}\right)
$$

Step 3. After the holonomic evolution has been completed, we read out $\Phi_{M}$ from the state of the probe qubit. Tracing out the system and ancilla in Eq. (9), the reduced state for the probe qubit is

$\rho_{P}=\frac{1}{2}\left(\cdot+\operatorname{Re}\left(\left\langle\Psi_{\theta(0)} \mid \Psi_{\theta\left(t_{f}\right)}\right\rangle\right) \sigma_{x}+\operatorname{Im}\left(\left\langle\Psi_{\theta(0)} \mid \Psi_{\theta\left(t_{f}\right)}\right\rangle\right) \sigma_{y}\right)$.

Thus, by measuring the expectation values $\left\langle\sigma_{x}\right\rangle$ and $\left\langle\sigma_{y}\right\rangle$ (green block of Fig. 2), we can retrieve $\Phi_{M}$ in the form

$$
\begin{aligned}
\Phi_{M} & =\arg \left[\left\langle\sigma_{x}\right\rangle+i\left\langle\sigma_{y}\right\rangle\right]= \\
& =\arg \left[\left\langle\Psi_{\theta(0)}\left|U_{S}\left(t_{f}\right) \otimes U_{A}\left(t_{f}\right)\right| \Psi_{\theta(0)}\right\rangle\right] .
\end{aligned}
$$

In Fig. 3 we present the results of phase measurements performed on the IBM Quantum Experience platform, ${ }^{56}$ using three transmon qubits coupled through co-planar waveguide resonators (see Methods). In Fig. 3a, we show the measurement of the Uhlmann phase $\Phi_{U}$ for different values of the mixedness parameter $r$, where we set $M=0.2$ and $p_{a}=p_{r}$, i.e., fulfilling the parallel transport condition. The critical jump from $\Phi_{U}=\pi$ (topological) to $\Phi_{U}=0$ (trivial) is clearly observed following the previous protocol.

Additionally, we can check whether the Uhlmann parallel transport condition is satisfied at every time interval during the experiment. By partitioning the closed trajectory in small time steps $\delta t$, the relative phase between the state at time $n \delta t$ and at $(n+1) \delta t$ must be close to zero if the condition is fulfilled. This is the case in the experiment as shown in Fig. 3b. During the state preparation (Step 1 ), we need to include two additional single qubit rotations $R_{y}^{a_{1}^{\text {not }}}$ and $R_{y}^{a^{n o t}}$ acting on the system and ancilla qubits respectively, where $a_{1}^{n \delta t}=2 I_{0}^{n \delta t}$ and $a_{2}^{n \delta t}=p_{r} a_{1}^{n \delta t}$. These two unitaries make the entangled state between system and ancilla evolve until the state $\left|\Psi_{\text {not }}\right\rangle$ is reached. In Step 2 , the state evolves to $\left|\Psi_{(n+1) \delta t}\right\rangle$ conditional to the state of the probe $P$. The 

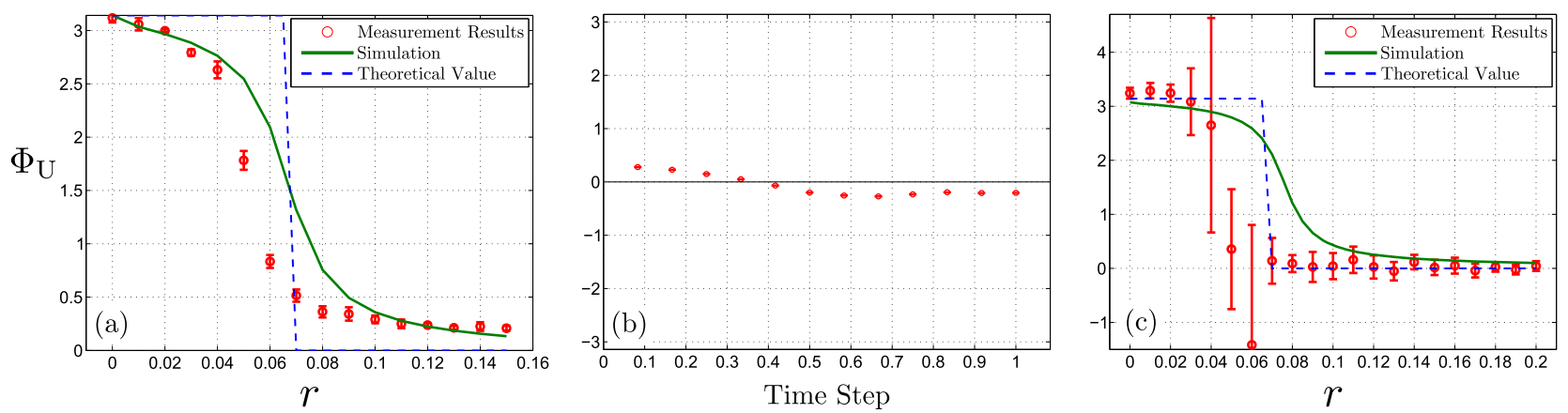

Fig. 3 Experimental results for the Uhlmann phase $\Phi_{U}$ as a function of the mixedness parameter $r$, defined in Eq. (1), or the time step in the holonomy. The red dots with error bars represent the experimental measurements, the dashed blue line is the theoretical value $\left(r_{c} \approx 0.067\right)$ and the green solid line accounts for a simulation based on an error model (see Methods). In a, we take $M=0.2$ and measure $\Phi_{\cup}$ as a function of the mixedness $r$ using $p_{a}=p_{r}$, i.e., fulfilling the parallel transport condition. We can clearly see the critical jump from $\Phi_{U}=\pi$ (topological) to $\Phi_{\mathrm{U}}=0$ (trivial). In $\mathbf{b}$, we plot, for $M=0.2, r=0.02$ and $t_{\mathrm{f}}=1$, the relative phase $\Phi$ between adjacent states for small time steps $\delta t=0.1$, checking

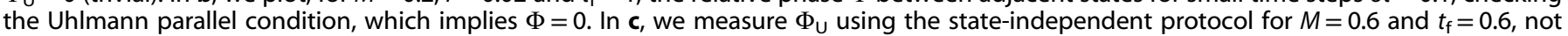
assuming prior knowledge of the mixedness $r$. The topological transition is clearly appreciable despite the presence of experimental imperfections. The experimental accuracy in $r$ is about 0.01

measurement scheme (Step 3) to retrieve the relative phase in Fig. $3 \mathrm{~b}$ remains the same. Technical details are described in the Supplementary Note 3 . We have included a simulation-green solid line in Fig. 3-based on experimental imperfections, mainly finite coherence time $(\sim 50 \mu \mathrm{s})$ and spurious terms accounting for certain type of electromagnetic crosstalk between qubits. A more detailed description of the error model is given in Methods.

\section{State-independent protocol}

The application of $U_{\mathrm{S}}(t)$ and $U_{\mathrm{A}}(t)$ with $p_{a}=p_{r}$ to the purification $\left|\Psi_{\theta(t)}\right\rangle$ implements the Uhlmann parallel transport and hence $\Phi_{M}$ $=\Phi_{\mathrm{U}}$. However, this would imply some knowledge about the mixedness parameter $r$ beforehand, which is not always possible. Hence, we present a modification of the previous protocol to measure the topological Uhlmann phase without prior knowledge of the state $\rho$ and its mixedness parameter $r$.

Firstly, we fix $\theta(t)=2 \pi t$ and consider open holonomies $\frac{1}{2}<t_{f}<1$ covering more than one half of the complete path. No previous knowledge of the state is assumed to perform the evolution. Hence, the ancillary weight $p_{a}$ can be different than $p_{r}$ in Eq. (2), but still satisfying $0 \leq p_{a} \leq 1$. From Eq. (7), the overlap $\left\langle\Psi_{\theta=0} \mid \Psi_{\theta=2 \pi t_{f}}\right\rangle$ is always real and thus the phase $\Phi_{M}$ is either 0 or $\pi$, depending on both the weight $p_{r}$ associated to the state $\rho_{\theta}$ [Eq. (1)] and the ancillary weight $p_{a}$.

We aim to find an $r$-independent value for $p_{a}$ such that the observed phase $\Phi_{M}$ takes on the same value as the Uhlmann phase for a Hamiltonian with the form of (3). By studying $\Phi_{M}$ as a function of the applied $p_{a}$, we conclude that if we tune the ancillary weight

$p_{a}=p_{T}:=\frac{-1}{l_{0}^{t_{f}}} \arctan \left(\frac{2}{\tan \left(I_{0}^{t_{f}}\right)}\right)$,

the value of the observed phase $\Phi_{M}\left(p_{a}=p_{T}\right)$ coincides with the topological Uhlmann phase $\Phi_{U}$. Algebraic details are provided in Methods.

Note that there is an intuitive reason why we can get topological information out of a phase associated to a open path longer than one half of a non-trivial topological loop. Indeed, $h(t)$ is symmetric around $t=\frac{1}{2}$. Then, once we have covered one half of the path, we know about the topology of the whole system thanks to this symmetry. Therefore, even an open path for $\frac{1}{2}<t_{f}<1$ can be considered as global.

In terms of the experimental protocol, we only need to modify Step 2 by fixing $p_{a}=p_{T}$ for the unitary $U_{\mathrm{A}}(t)$. In Fig. $3 c$, we present the results for the state-independent protocol recovering the topological Uhlmann phase without prior knowledge of the state, for $M=0.6$ and $t_{\mathrm{f}}=0.6$. These are qualitatively the same as in Fig. $3 a$, but the state-independent protocol is more sensitive to errors mainly around the transition point. The mismatch between experiment and simulations is most likely caused by small calibration-dependent systematic errors in the cross-resonance gates.

\section{DISCUSSION}

We have successfully measured the topological Uhlmann phase, originally proposed in the context of Tls and superconductors, making use of ancilla-based protocols. The experiment is realized within a minimal quantum simulator consisting of three superconducting qubits. We have exploited the quantum simulator to realize a controlled coupling of the system to an environment represented by the ancilla degrees of freedom. Moreover, we have proposed and tested a state-independent protocol that allows us to classify states of topological systems according to the Uhlmann measure. To our knowledge, this is the first time that a noise/ temperature-induced topological transition in a quantum phase is observed. Recently, these transitions have been addressed in connection to new thermodynamical properties of these systems. ${ }^{57}$ The fact that these effects can be experimentally observed opens the possibility for the search of warm topological matter in the lab. Due to the intrinsic geometric character of the Uhlmann phase, our results may find application in generalizations of holonomic quantum protocols for general, possibly mixed, states.

In addition, an increase of experimental resources such as the number of qubits, the speed and fidelity of the quantum gates, etc. will allow us to study additional topological phenomena with superconducting qubits. In particular, by including interactions in the model Hamiltonian we can test different features: quantum simulations of thermal topological transitions in 2D TIs and TSCs, the interplay between noise and interactions within a topological phase, etc. These effects can be achieved since a system with more interacting qubits can be mapped onto models for interacting fermions with spin. ${ }^{15}$ Further details can be found in the Supplementary Note 5. Although such a proposal would be experimentally more demanding, it represents a clear outlook that would need precise controllability of more qubits and the ability to perform more gates with high fidelity. 


\section{METHODS}

Superconducting qubit realization of a controllable uhlmann phase

The experiments on the topological Uhlmann phase have been realized on the IBM Quantum Experience (ibmqx2), ${ }^{56}$ a quantum computing platform with online user-access based on five fixed-frequency transmon-type qubits coupled via co-planar waveguide resonators. We have used three qubits, qubit Q0 as the probe qubit, Q1 as the system qubit and Q2 as the ancilla qubit. This choice is motivated by the connectivity required for the measurement protocol and the superior $T_{1}$ and $T_{2}$ times of this set of qubits when compared to the set $\{Q 2, Q 3, Q 4\}$ at the time of the experiment. We have used the open-source python SDK QISKit (https:// www.qiskit.org) to program the quantum computer and retrieve the data. The explicit quantum algorithm to measure the expectation values of $\sigma_{x}$ and $\sigma_{y}$ is provided in Supplementary Note 4 using the OPENQASM intermediate representation (https://github.com/QISKit/openqasm). The phase is then extracted from the measured data by evaluating $\Phi_{M}=\arg \left(\left\langle\sigma_{x}\right\rangle-i\left\langle\sigma_{y}\right\rangle\right)$.

For all experiments we have measured 8192 repetitions providing a single value for the phase. For the measurement of the topological Uhlmann phase (Fig. 3a) we vary the initial mixedness of the system state $r$ by setting the rotation angle $\gamma=2 \arccos (\sqrt{1-r})$. The transport of the state according to UhImann's parallel transport condition is set by the value $\beta_{1}=I_{f}(0,1)=\pi$ for $M<1$ and $\beta_{2}=p_{a} I_{f}(0,1)=2 \pi \sqrt{r(1-r)}$, as defined in Eq. (7). The energy relaxation times of the qubits are $\left\{T_{1}^{\mathrm{Q0}}, T_{1}^{\mathrm{Q} 1}, T_{1}^{\mathrm{Q} 2}\right\}=\{45 \mu \mathrm{s}, 31 \mu \mathrm{s}, 46 \mu \mathrm{s}\}$ and the decoherence times $\left\{T_{2}^{\mathrm{Q0}}, T_{2}^{\mathrm{Q1}}, T_{2}^{\mathrm{Q} 2}\right\}=\{40 \mu \mathrm{s}, 27 \mu \mathrm{s}, 80 \mu \mathrm{s}\}$ as stated in the calibration data.

For the state-independent protocol [Fig. 3c, main text] we set $M=0.6$ and the final time $t_{\mathrm{f}}=0.6$. The system is rotated about $\beta_{1}=I_{0}^{0.6}=2.18537$ and $\beta_{2}=p_{T} l_{0}^{0.6}=0.954407$. In this measurement energy relaxation and decoherence times are $\left\{T_{1}^{\mathrm{Q0}}, T_{1}^{\mathrm{Q1}}, T_{1}^{\mathrm{Q2}}\right\}=\{41 \mu \mathrm{s}, 52 \mu \mathrm{s}, 62 \mu \mathrm{s}\}$ and $\left\{T_{2}^{Q 0}, T_{2}^{Q 1}, T_{2}^{Q 2}\right\}=\{31 \mu \mathrm{s}, 37 \mu \mathrm{s}, 87 \mu \mathrm{s}\}$. Note, that here the error bars are larger as compared to the state-dependent measurement described above, because the expectation values $\left\langle\sigma_{x}\right\rangle$ and $\left\langle\sigma_{y}\right\rangle$ are closer to zero leading to larger statistical errors in the phase. Also, we notice a systematic offset of $\bar{\sigma}_{y}=0.098 \pm 0.014$ from the expected value $\left\langle\sigma_{y}\right\rangle_{\text {th }}=0$. Here, $\bar{\sigma}_{y}$ is the average over all $r$ values and repetitions. This offset is subtracted from the phase data $\Phi_{M}=\arg \left[\left\langle\sigma_{x}\right\rangle-i\left\langle\left\langle\sigma_{y}\right\rangle-\bar{\sigma}_{y}\right\rangle\right]$ and the result is plotted in Fig. $3 c$. We consider accumulated phase shifts during two-qubit operations as the main reason for this mismatch. We have also noticed that this value changes for different calibrations of the IBM Quantum Experience and when taking different sets of qubits.

Finally, for the measurement of the parallel transport condition we modify the algorithm to prepare the intermediate state $\left|\Psi_{\theta(n \delta t)}\right\rangle$ by applying $U_{S / A}(n \delta t)$ to system and ancilla qubit. For the measurement of the Uhlmann phase, the same circuit as above is used to obtain a state evolution $\left|\Psi_{\theta(n \delta t)}\right\rangle \rightarrow\left|\Psi_{\theta((n+1) \delta t)}\right\rangle$. The complete protocol to measure the parallel transport condition is shown in the Supplementary Fig. 1. In the experiment, we choose $M=0.2$ and $r=0.02$ to stay within the topological sector. The mixedness angle evaluates to $\gamma=2 \arccos (\sqrt{0.95})=0.2838$. The angles for the intermediate state preparation are determined by $a_{1}(n)=I_{0}^{n \delta t}$ and $a_{2}(n)=p_{r} I_{0}^{n \delta t}=2 \sqrt{r(1-r)} I_{0}^{n \delta t}=0.28 I_{0}^{n \delta t}$, the evolution from $n \delta t$ to $(n+1) \delta t$ is determined by the angles $\beta_{1}(n)=I_{n \delta t}^{(n+1) \delta t}$ and $\beta_{2}(n)=p_{r} r_{n \delta t}^{(n+1) \delta t}=0.28 I_{n \delta t}^{(n+1) \delta t}$. The recorded data shown in Fig. 3b, main text, shows that the measured phase difference $\left\langle\Phi_{M}(n \delta t)\right\rangle=-0.07 \pm 0.2$ is zero within the statistics. However, the residuals do not follow a normal distribution which hints at systematic gate errors instead of stochastic errors.

State-independent derivation

The derivation of the value for $p_{T}$ [Eq. (12)] is as follows. From Eq. (7) we find the value $p_{a}=p_{a}^{c}$ (where the superindex $c$ stands for critical) at which $\Phi_{\mathrm{M}}$ goes abruptly from $\pi$ to 0 as a function of $p_{r}$ and $I_{\mathrm{f}}$

$p_{a}^{c}=\frac{-1}{l_{0}^{t_{f}}} \arctan \left(\frac{1}{p_{r} \tan \left(l_{0}^{t_{f}}\right)}\right)$.

If we set $\frac{1}{2}<t_{f}<1$, then $p_{a}^{c}$ is a monotonically decreasing function of $p_{r}$,

$\frac{\partial p_{a}^{c}}{\partial p_{r}}=\frac{\tan \left(I_{0}^{t_{f}}\right)}{l_{0}^{t_{f}}\left[1+p_{r}^{2} \tan ^{2}\left(I_{0}^{t_{f}}\right)\right]}<0$.
If $M>1$, then $-\pi / 2<l_{0}^{t_{f}}<\pi / 2$, which from Eq. (7) implies that $\Phi_{M}=0$ for any value of $p_{r}$ and $p_{a}$. Hence, for the trivial case $M>1$, there is no critical value $p_{a}^{c}$ and $\Phi_{M}=0$ always. This maps $\Phi_{M}$ to the Uhlmann phase $\Phi_{U}$ at least for this case. On the contrary, if $M<1$, then $\pi / 2<t_{0}^{t_{f}}<\pi$ which implies $\tan \left(I_{0}^{t_{f}}\right)<0$. Since $0<p_{r}<1$, then $-\arctan \left(\frac{1}{p_{r} \tan \left(I_{0}^{t_{f}}\right)}\right)<\pi / 2$. Thus, there is always a solution of Eq. (13) with $0<p_{a}^{c}<1$ for any $p_{r}$. As discussed in the main text, the state $\rho_{\theta}$ in Eq. (1) is topological in the Uhlmann sense $\Phi_{U}=\pi$, only if $M<1$ and $p_{r}<0.5$.

Now, we define $p_{T}:=p_{a}^{c}\left(p_{r}=0.5\right)$ using Eq. (13). Note that the true $p_{r}$ of the system is unknown as we have assumed no knowledge of the state. Nevertheless, if $p_{r}>0.5$, then its associated critical value [from Eq. (13)] is $p_{a}^{c}<p_{T}$. This means that by applying $U_{\mathrm{A}}$ with $p_{a}=p_{\mathrm{T}}$ and measuring the associated phase $\Phi_{M}$ we can extract the following conclusions:

- If we measure $\Phi_{M}\left(p_{T}\right)=0$, the system is within a trivial phase $\left(\Phi_{U}=0\right)$. Because this implies $p_{a}^{c}<p_{T}$ and hence $p_{r}>0.5\left(\Phi_{U}=0\right)$, as we have proven that $p_{a}^{c}$ always decreases with $p_{r}$.

- If we measure $\Phi_{\mathrm{M}}\left(p_{T}\right)=\pi$, the system is in a topological phase $\left(\Phi_{U}=\pi\right)$. Because in that case $p_{a}^{c}>p_{T}$ and then $p_{r}<0.5\left(\Phi_{U}=\pi\right)$.

Hence, we have just proven that $\Phi_{\mathrm{M}}\left(p_{\mathrm{T}}\right)=\Phi_{\mathrm{U}}$.

Error simulation

The detrimental effect of experimental errors is modeled by means of a Liouvillian term $\mathcal{L}_{\text {error, }}$ so that the Liouvillian $\mathcal{L}_{0}$, accounting for the idealized dynamics, is in fact substituted by $\mathcal{L}_{0}+\mathcal{L}_{\text {error }}$. Specifically, if a gate is performed during a time $\tau$ via a Hamiltonian $H_{0}$, i.e., $U_{\text {gate }}=e^{-i H_{0} \tau}$, we substitute

$e^{-i H_{0} \tau} \rho e^{i H_{0} \tau} \equiv e^{\mathcal{L}_{0} \tau} \rho \rightarrow e^{\left(\mathcal{L}_{0}+\mathcal{L}_{\text {error }}\right) \tau} \rho$.

This error Liouvillian includes typical sources of imperfections: a) a residual IX term during the cross-resonance $Z X 90$ gate in the implementation of the CNOTs, $H_{Z X}=m I X+\mu Z X_{i}^{58-60}$ b) spontaneous emission and dephasing terms $\mathcal{L}_{-}(\rho)=\gamma_{-}\left(\sigma_{-} \rho \sigma_{+}-\frac{1}{2}\left\{\sigma_{+} \sigma_{-}, \rho\right\}\right) \quad$ and $\mathcal{L}_{z}(\rho)=\gamma_{z}\left(\sigma_{z} \rho \sigma_{z}-\rho\right)$, respectively.

We have accommodated the values of $\gamma_{-}$and $\gamma_{z}$ to the characteristic longitudinal and transverse relaxation times of $T_{1}=51 \mu \mathrm{s}$ and $T_{2}=51 \mu \mathrm{s}$ reported by the IBM Quantum Experience calibration team the day of the measurements. The residual IX strength has been taken to be about $m \sim$ $0.4 \mathrm{MHz}$. In addition, we consider $\tau_{2 \pi} \sim 200 \mathrm{~ns}$ and $\tau_{Z \times 90} \sim 600 \mathrm{~ns}$ as characteristic times for a $2 \pi$-rotation on a single qubit and the ZX90 gates, respectively. Waiting times of $5 \mathrm{~ns}$ after a single qubit gate and $40 \mathrm{~ns}$ after a ZX90 gate are also included.

In Fig. 3, we plot the result of the simulation including these experimental imperfections together with the experimental measurements of the topological Uhlmann phase $\Phi_{\mathrm{U}}$. Despite the errors, the topological transition is clearly noticed.

Data availability

All relevant data are available from the authors on reasonable request.

\section{ACKNOWLEDGEMENTS}

M.A.M.D., A.R. and O.V. thank the Spanish MINECO grant FIS2012-33152, a "Juan de la Cierva-Incorporación" reseach contract, the CAM research consortium QUITEMAD+ S2013/ICE-2801, the U.S. Army Research Office through grant W911NF-14-1-0103, Fundación Rafael del Pino, Fundación Ramón Areces, and RCC Harvard. S.G., A.W. and S.F. acknowledge support by the Swiss National Science Foundation (SNF, Project 150046).

\section{AUTHOR CONTRIBUTIONS}

O.V., A.R. and M.A.M.D. conceived and developed the theoretical project. S.G., A.W. and S.F. devised the experiment. S.F. carried out the experimental measurements and analyzed the data with input from the theoretical teams. All authors wrote the manuscript.

\section{ADDITIONAL INFORMATION}

Supplementary information accompanies the paper on the npj Quantum Information website (https://doi.org/10.1038/s41534-017-0056-9).

Competing interests: The authors declare no competing financial interests. 
Publisher's note: Springer Nature remains neutral with regard to jurisdictional claims in published maps and institutional affiliations.

\section{REFERENCES}

1. Hasan, M. Z. \& Kane, C. L. Rev. Mod. Phys. 82, 3045 (2010)

2. Qi, X.-L. \& Zhang, S.-C. Rev. Mod. Phys. 83, 1057 (2011).

3. Bernevig, B. A. \& Hughes, T. L. Topological Insulators and Topological Superconductors. (Princeton University Press, NJ, 2013).

4. Bernevig, B. A., Hughes, T. L. \& Zhang, S.-C. Quantum spin Hall effect and topological phase transition in HgTe quantum wells. Science 314, 1757-1761 (2006)

5. Koenig, M. et al. Quantum spin hall insulator state in HgTe quantum wells. Science 318, 766-770 (2007).

6. Hsieh, T. H. et al. Topological crystalline insulators in the SnTe material class. Nat. Commun. 3, 982 (2012).

7. Dziawa, P. et al. Topological crystalline insulator states in $\mathrm{Pb}_{1-x} \mathrm{Sn}_{\mathrm{x}} \mathrm{Se}$. Nat. Mater. 11, 1023-1027 (2012).

8. $\mathrm{Xu}, \mathrm{S}$. et al. Discovery of a Weyl fermion semimetal and topological Fermi arcs. Science 349, 613-617 (2015).

9. Atala, M. et al. Direct measurement of the Zak phase in topological Bloch bands. Nat. Phys. 9, 795 (2013).

10. Jotzu, G. et al. Experimental realization of the topological Haldane model with ultracold fermions. Nature 515, 237-240 (2014)

11. Duca, L. et al. An Aharonov-Bohm interferometer for determining Bloch band topology. Science 347, 288 (2015).

12. Li, T. et al. Bloch state tomography using Wilson lines. Science 352, 1094 (2016).

13. Flurin, E. et al. Observing Topological Invariants Using Quantum Walks in Superconducting Circuits. Phys. Rev. X 7, 031023 (2017).

14. Schroer, M. D. et al. Measuring a Topological Transition in an Artificial Spin-1/2 System. Phys. Rev. Lett. 113, 050402 (2014).

15. Roushan, P. et al. Observation of topological transitions in interacting quantum circuits. Nature 515, 241-244 (2014).

16. Leek, P. J. et al. Observation of Berry's Phase in a Solid-State Qubit. Science 318, 1889-1892 (2007).

17. Nielsen, M. A. \& Chuang, I. L. Quantum Computation and Quantum Information. (Cambridge University Press, Cambridge, 2000).

18. Galindo, A. \& Martin-Delgado, M. A. Information and Computation: Classical and Quantum Aspects. Rev. Mod. Phys. 74, 347 (2002).

19. Berry, M. V. Quantal phase factors accompanying adiabatic changes. Proc. R. Soc. A 392, 45 (1984).

20. Shapere, A. \& Wilczek, F. Geometric Phases in Physics. (World Scientific, Singapore, 1989).

21. Zak, J. Berry's phase for energy bands in solids. Phys. Rev. Lett. 62, 2747 (1989).

22. Viyuela, O., Rivas, A. \& Martin-Delgado, M. A. Thermal Instability of Protected End States in a 1-D Topological Insulator. Phys. Rev. B 86, 155140 (2012).

23. Rivas, A., Viyuela, O. \& Martin-Delgado, M. A. Density-matrix Chern insulators: Finite-temperature generalization of topological insulators. Phys. Rev. B 88, 155141 (2013).

24. Mazza, L., Rizzi, M., Lukin, M. D. \& Cirac, J. I. Robustness of quantum memories based on Majorana zero modes. Phys. Rev. B 88, 205142 (2013).

25. Bardyn, C.-E. et al. Topology by dissipation New. J. Phys. 15, 085001 (2013).

26. van Nieuwenburg, E. P. L. \& Huber, S. D. Classification of Mixed State Topology in One Dimension. Phys. Rev. B 90, 075141 (2014).

27. Shen, H. Z., Wang, W. \& Yi, X. X. Hall conductance and topological invariant for open systems. Sci. Rep. 4, 6455 (2014).

28. Albert, V. V., Bradlyn, B., Fraas, M. \& Jiang, L. Phys. Rev. X 6, 041031 (2016).

29. Dehghani, H., Oka, T. \& Mitra, A. Dissipative Floquet topological systems. Phys. Rev. B 90, 195429 (2014).

30. Hu, Y., Cai, Z., Baranov, M. \& Zoller, P. Majoranas in Noisy Kitaev Wires. Phys. Rev. B 92, 165118 (2015).

31. Linzner, D., Wawer, L., Grusdt, F. \& Fleischhauer, M. Reservoir-induced Thouless pumping and symmetry-protected topological order in open quantum chains. Phys. Rev. B 94, 201105 (2016).

32. Claeys, P. W., De Baerdemacker, S. \& Van Neck, D. Read-Green resonances in a topological superconductor coupled to a bath. Phys. Rev. B 93, 220503(R) (2016).

33. Lemini, F., Rossini, D., Fazio, R., Diehl, S. \& Mazza, L. Dissipative topological superconductors in number-conserving systems. Phys. Rev. B 93, 115113 (2016).

34. Bardyn, C.-E., Wawer, L., Altland, A., Fleischhauer, M., Diehl, S. Probing the topology of density matrices, arXiv: 1706.02741 (2017).

35. Viyuela, O., Rivas, A. \& Martin-Delgado, M. A. Uhlmann Phase as a Topological Measure for One-Dimensional Fermion Systems. Phys. Rev. Lett. 112, 130401 (2014).
36. Viyuela, O., Rivas, A. \& Martin-Delgado, M. A. Two-Dimensional Density-Matrix Topological Fermionic Phases: Topological Uhlmann Numbers. Phys. Rev. Lett. 113, 076408 (2014).

37. Huang, Z. \& Arovas, D. P. Topological Indices for Open and Thermal Systems Via Uhlmann's Phase. Phys. Rev. Lett. 113, 076407 (2014).

38. Viyuela, O., Rivas, A. \& Martin-Delgado, M. A. Symmetry-protected Topological Phases at Finite Temperature. 2D Mater. 2, 034006 (2015).

39. Uhlmann, A. Parallel transport and "quantum holonomy" along density operators. Rep. Math. Phys. 24, 229 (1986).

40. Sjöqvist, E. et al. Geometric Phases for Mixed States in Interferometry. Phys. Rev. Lett. 85, 2845 (2000).

41. Ericsson, M., Pati, A. K., Sjöqvist, E., Brännlund, J. \& Oi, D. K. L. Mixed State Geometric Phases, Entangled Systems, and Local Unitary Transformations. Phys. Rev. Lett. 91, 090405 (2003).

42. Åberg, J., Kult, D., Sjöqvist, E. \& Oi, D. K. L. Operational approach to the Uhlmann holonomy. Phys. Rev. A. 75, 032106 (2007).

43. Zhu, J. et al. Experimental demonstration of a unified framework for mixed-state geometric phases EPL 94, 20007 (2011).

44. Budich, J. C. \& Diehl, S. Topology of density matrices. Phys. Rev. B 91, 165140 (2015).

45. Andersson, O., Bengtsson, I., Ericsson, M. \& Sjöqvist, E. Geometric phases for mixed states of the Kitaev chain. Philos. Trans. R. Soc. A 374, 20150231 (2016).

46. Mera, B., Vlachou, C., Paunković, N. \& Vieira, V. R. Boltzmann-Gibbs states in topological quantum walks and associated many-body systems: Fidelity and Uhlmann parallel transport analysis of Phase Transitions. J. Phys. A: Math. Theor. 50, 365302 (2017).

47. Mera, B., Vlachou, C., Paunković, N. \& Vieira, V. R. The Uhlmann connection in fermionic systems undergoing phase transitions, Phys. Rev. Lett. 119, 015702 (2017).

48. Schoelkopf, R. J. \& Girvin, S. M. Wiring up quantum systems. Nature 451, 664-669 (2008).

49. Barends, R. et al. Digital quantum simulation of fermionic models with a superconducting circuit. Nat. Commun. 6, 7654 (2015).

50. Salathé, Y. et al. Digital Quantum Simulation of Spin Models with Circuit Quantum Electrodynamics. Phys. Rev. X 5, 021027 (2015).

51. Schnyder, A. P., Ryu, S., Furusaki, A. \& Ludwig, A. W. W. Classification of topological insulators and superconductors in three spatial dimensions. Phys. Rev. B 78, 195125 (2008)

52. Kitaev, A. Periodic table for topological insulators and superconductors. AIP Conf. Proc. 1134, 22 (2009).

53. Koch, J. et al. Charge-insensitive qubit design derived from the Cooper pair box. Phys. Rev. A 76, 042319 (2007)

54. Strauch, F. W. et al. Quantum logic gates for coupled superconducting phase qubits. Phys. Rev. Lett. 91, 167005 (2003).

55. Chow, J. M. et al. Simple All-Microwave Entangling Gate for Fixed-Frequency Superconducting Qubits. Phys. Rev. Lett. 107, 080502 (2011).

56. IBM. IBM Quantum Experience. http://research.ibm.com/ibm-qx/.

57. Kempkes, S. N., Quelle, A. \& Smith, C. M. Universalities of thermodynamic signatures in topological phases. Sci. Rep. 6, 38530 (2016).

58. Córcoles, A. D. et al. Process verification of two-qubit quantum gates by randomized benchmarking. Phys. Rev. A. 87, 030301(R) (2013).

59. Chow, J. M. et al. Implementing a strand of a scalable fault-tolerant quantum computing fabric. Nat. Commun. 5, 4015 (2014).

60. Sheldon, S., Magesan, E., Chow, J. M. \& Gambetta, J. M. Procedure for systematically tuning up cross-talk in the cross-resonance gate. Phys. Rev. A. 93, 060302 (2016).

Open Access This article is licensed under a Creative Commons Attribution 4.0 International License, which permits use, sharing, adaptation, distribution and reproduction in any medium or format, as long as you give appropriate credit to the original author(s) and the source, provide a link to the Creative Commons license, and indicate if changes were made. The images or other third party material in this article are included in the article's Creative Commons license, unless indicated otherwise in a credit line to the material. If material is not included in the article's Creative Commons license and your intended use is not permitted by statutory regulation or exceeds the permitted use, you will need to obtain permission directly from the copyright holder. To view a copy of this license, visit http://creativecommons. org/licenses/by/4.0/.

(c) The Author(s) 2018 